\title{
Factores que influyen en la adherencia de los trabajadores de la salud a las medidas de prevención y control de infecciones respiratorias
}

\author{
Factors influencing health workers' adherence to respiratory infection prevention and control measures
}

\section{Comentado de:}

Houghton C, et al. Cochrane Database Syst Rev. 2020, 4(4):CD013582. PMID: $32315451^{1}$

\section{Objetivo}

Describir los factores que influyen en la adherencia de los trabajadores de la salud a las guías de prevención y control de infecciones respiratorias.

\section{Diseño}

Síntesis de evidencia cualitativa.

\section{Población}

Personal de salud con responsabilidad directa en la atención de pacientes.

\section{Intervención}

Guías institucionales, nacionales o internacionales para prevención y control de infecciones respiratorias dentro de las cuales se incluyeron la enfermedad por coronavirus 2019 (COVID-19), el síndrome respiratorio agudo severo (SARS, por sus iniciales en inglés), el síndrome respiratorio de Medio Oriente (MERS, por sus iniciales en inglés), la tuberculosis y la influenza. Estas guías contemplan medidas como higiene de manos, uso de equipos de protección personal (EPP), controles administrativos, entre otras.

\section{Métodos}

Los autores realizaron una búsqueda bibliográfica de estudios cualitativos que abordaban la temática de interés. Utilizaron un modelo teórico preexistente como marco de referencia para extraer, analizar y sintetizar la información de los estudios primarios. Aplicaron la herramienta GRADE-CERQual para evaluar el grado de confianza en los hallazgos de la revisión. Se trató de una revisión rápida en el contexto de la pandemia de COVID-19, lo que permitió acelerar el proceso de realización de la revisión omitiendo ciertos pasos metodológicos.

\section{Resultados}

Los autores encontraron 36 estudios elegibles de los cuales seleccionaron 20 para su análisis, sobre la base de criterios de relevancia (incluyeron todos los estudios sobre coronavirus), riqueza conceptual y diversidad geográfica. Los resultados del análisis se resumieron en 26 hallazgos clave, comprendidos en tres categorías. En la Tabla 1 se presenta un resumen de los hallazgos más relevantes.

Tabla 1. Resumen de hallazgos seleccionados de la revisión: factores que influyen en la adherencia de los trabajadores de la salud a las medidas de prevención y control de infecciones respiratorias propuestas por las guías de práctica clínica

\begin{tabular}{|c|c|}
\hline Factores & Categorías destacadas \\
\hline Individuales & $\begin{array}{l}\text { Conocimientos, creencias y actitudes individuales. Se señalan como facilitadores el temor al } \\
\text { contagio tanto individual como de colegas, familiares y amigos, y la creencia en la efectividad de las } \\
\text { medidas. Como barrera la preocupación por estigmatizar o atemorizar a los pacientes, en particular } \\
\text { cuando éstos eran niños. La cultura de seguridad en el trabajo y la presión de pares puede actuar } \\
\text { como facilitador o barrera. } \\
\text { Incomodidad de los EPP. Se señala como una barrera. }\end{array}$ \\
\hline Ambientales & $\begin{array}{l}\text { Espacio físico. Son facilitadores la presencia de una infraestructura adecuada (habitaciones indivi- } \\
\text { duales, espacios ventilados), la implementación de medidas prácticas para regular el flujo de perso- } \\
\text { nas y evitar la congestión y la disponibilidad de espacios e insumos para la higiene de manos. } \\
\text { Disponibilidad de los EPP. El acceso o falta de acceso a los mismos son factores clave que influyen } \\
\text { en la adherencia a las medidas preventivas. }\end{array}$ \\
\hline Organizacionales & $\begin{array}{l}\text { Comunicación de las guías. Necesidad de directivas claras, fácilmente accesibles, adaptadas al } \\
\text { contexto de la atención. } \\
\text { Disponibilidad de programas de entrenamiento } \\
\text { Clima de seguridad. Son facilitadores el apoyo y reconocimento por parte de las autoridades (moral, } \\
\text { económico) y la valoración de las necesidades de los trabajadores. Como barrera se describe un } \\
\text { aumento en la carga de trabajo que puede llevar al agotamiento si no se toman medidas compensa- } \\
\text { torias. }\end{array}$ \\
\hline
\end{tabular}




\section{Conclusiones}

Los trabajadores de la salud señalan varios factores que influyen en su capacidad y disposición para seguir las pautas de prevención y control de infecciones cuando asisten a pacientes con enfermedades infecciosas respiratorias. Estos incluyen factores vinculados a la guía de práctica misma y cómo esta se comunica, el apoyo de los gestores de salud, la cultura del lugar de trabajo, la capacitación, el espacio físico, la accesibilidad y la confianza en el EPP y el deseo de brindar una buena atención al paciente. La revisión también destaca la importancia de incluir a todo el personal de la institución, incluido el personal de apoyo, al implementar las pautas de prevención y control de infecciones.

Fuentes de financiamiento/Conflicto de interés de los autores: Ninguno conocido.

\section{Comentario}

Los estudios cualitativos nos ayudan a comprender mejor las experiencias y las percepciones de las personas (en este caso, los trabajadores de la salud) sobre determinadas temáticas, como problemas de salud o tratamientos, nos permite complementar la información proveniente de estudios cuantitativos y resulta de gran importancia para la toma de decisiones en el ámbito sanitario ${ }^{2}$. Las síntesis de evidencia cualitativa son un intento de recopilar, analizar y sintetizar en forma sistemática este tipo de información.

Un aspecto metodológico clave de este tipo de revisiones es determinar en qué medida sus hallazgos o conclusiones son confiables o representativos del fenómeno en cuestión. Con esta finalidad los autores utilizan una herramienta denominada GRADE CERQual, la que toma en consideración cuatro dominios: las limitaciones metodológicas de los estudios primarios, su relevancia con respecto a los objetivos de la revisión, la coherencia entre los hallazgos y los resultados de los estudios primarios y la calidad de los datos que sustentan cada hallazgo, incluyendo la riqueza conceptual y la descripción detallada del contexto en que se obtuvieron ${ }^{3}$.

Muchos de los elementos que señalan los autores en este artículo son fácilmente reconocibles y aplicables en el contexto actual, de modo que pueden servir como disparadores para la reflexión sobre nuestro trabajo diario como efectores de salud. Los puntos más destacados por médicos de familia que participaron de la discusión de este trabajo en ateneo bibliográfico en el Hospital Italiano de Buenos Aires fueron el temor de los profesionales al contagio, la incertidumbre en cuanto a la efectividad de las medidas preventivas, la importancia de contar con programas de entrenamiento y la necesidad de apoyo y reconocimiento por parte de las autoridades. Se señaló además la importancia de reconocer que, más allá de la pandemia actual de COVID-19, el riesgo de transmisión de enfermedades infecciosas está siempre presente en la práctica de las profesiones de la salud, particularmente en los entornos hospitalario y de urgencias. El análisis y la reflexión sobre la situación actual puede incentivar una mayor conciencia, tanto en los trabajadores como de la población general, de la relevancia de las medidas preventivas.

\section{Conclusiones del comentador}

Los factores que influyen en la adherencia del personal de la salud a las medidas de prevención de infecciones son múltiples, y la clasificación en determinantes individuales, ambientales e institucionales resulta útil para la reflexión constructiva sobre nuestra práctica cotidiana como personal de salud y el entorno en el cual la llevamos a cabo.

Sebastián Sguiglia [ Servicio de Medicina Familiar y Comunitaria, Hospital Italiano de Buenos Aires. sebastian.sguiglia@hospitalitaliano.org.ar ]

Sguiglia S Factores que influyen en la adherencia de los trabajadores de la salud a las medidas de prevención y control de infecciones respiratorias. Evid Actual Pract Ambul. 2020;23(2):e002068. Comentado de: Houghton C, et al. Barriers and facilitators to healthcare workers' adherence with infection prevention and control (IPC) guidelines for respiratory infectious diseases: a rapid qualitative evidence synthesis. Cochrane Database Syst Rev. 4(4):CD013582. PMID: 32315451

\section{Referencias}

1. Houghton C, Meskell P, Delaney H, et al. Barriers and Facilitators to Healthcare Workers' Adherence With Infection Prevention and Control (IPC) Guidelines for Respiratory Infectious Diseases: A Rapid Qualitative Evidence Synthesis. Cochrane Database Syst Rev. $2020 ; 4(4)$ :CD013582. Available from: 10.1002/14651858.CD013582.

2. Noyes J, Booth A, Cargo M, et al.. Chapter 21: Qualitative evidence. En: Higgins J, Thomas J. Cochrane Handbook for Systematic Reviews of Interventions. Version 6.0; 2019. Available from: https://training.cochrane.org/handbook/current.

3. Lewin S, Glenton C, Munthe-Kaas H, et al. Using Qualitative Evidence in Decision Making for Health and Social Interventions: An Approach to Assess Confidence in Findings from Qualitative Evidence Syntheses (GRADE-CERQual). PLoS Med. 2015;12(10):e1001895. Available from: 10.1371/journal.pmed.1001895 